\title{
A Distributed Camera System for Multi-Resolution Surveillance
}

\author{
Nicola Bellotto*, Eric Sommerlade*, Ben Benfold*, Charles Bibby*, Ian Reid*, \\ Daniel Roth ${ }^{\dagger}$, Carles Fernández ${ }^{\ddagger}$, Luc Van Gool ${ }^{\dagger}$ and Jordi Gonzàlez ${ }^{\ddagger}$ \\ *Active Vision Laboratory, University of Oxford, United Kingdom \\ http://www.robots.ox.ac.uk/ActiveVision \\ ${ }^{\dagger}$ Computer Vision Laboratory, ETH Zurich, Switzerland \\ Email: $\{$ droth,vangool $\} @$ vision.ee.ethz.ch \\ ${ }^{\ddagger}$ Computer Vision Centre, Bellaterra, Spain \\ Email: $\{$ perno,poal $\} @$ cvc.uab.es
}

\begin{abstract}
We describe an architecture for a multi-camera, multi-resolution surveillance system. The aim is to support a set of distributed static and pan-tilt-zoom (PTZ) cameras and visual tracking algorithms, together with a central supervisor unit. Each camera (and possibly pan-tilt device) has a dedicated process and processor. Asynchronous interprocess communications and archiving of data are achieved in a simple and effective way via a central repository, implemented using an SQL database.

Visual tracking data from static views are stored dynamically into tables in the database via client calls to the SQL server. A supervisor process running on the SQL server determines if active zoom cameras should be dispatched to observe a particular target, and this message is effected via writing demands into another database table.

We show results from a real implementation of the system comprising one static camera overviewing the environment under consideration and a PTZ camera operating under closed-loop velocity control, which uses a fast and robust level-set-based region tracker. Experiments demonstrate the effectiveness of our approach and its feasibility to multi-camera systems for intelligent surveillance.
\end{abstract}

\section{INTRODUCTION}

We are motivated by the desire to develop cognitive visual systems. In the context of surveillance, the theme of this paper, by cognitive we mean a system which can not only track targets, but identify them, and explain what is taking place, especially taking account of any possible causal relationships.

This paper describes a system which we hope will support work towards this goal. Here, we are primarily concerned with the low-level data acquisition processes, how these processes communicate, and also how any solution to these problems could be used in the context of top-down processing. To this end we describe an architecture for a multi-camera, multiresolution surveillance system. The proposed solutions and, in particular, the use of a central SQL database provide a reference application for the asynchronous communication between visual processes, as well as an efficient way to store large amounts of information for possible reasoning engines.

The system comprises a set of distributed static and PTZ cameras and visual tracking algorithms, together with a central supervisor unit. Each camera (and possibly pan-tilt device) has a dedicated process and processor. Asynchronous interprocess communications and archiving of data are achieved in a simple and effective way via a central repository, implemented using an SQL database. Visual tracking data from static views are stored dynamically into tables in the database via client calls to the SQL server. A supervisor process running on the SQL server determines if active zoom cameras should be dispatched to observe a particular target, and this message is effected via writing demands into another database table.

We show initial results from a real implementation of the system comprising a static and a PTZ camera, although the system can be easily extended to include more devices thanks to the modularity of our approach. The static camera runs a figure/ground segmentation-based tracker, while the PTZ camera operates under closed-loop velocity control using a fast and robust level-set-based region tracker. The goal of the system, as regulated by a supervisor process, is to keep track of all targets in a scene using the overview camera, and to acquire high-resolution, stabilised images of the faces of all agents in the scene using zoom cameras in closed loop tracking mode.

The remainder of the paper is organised as follows: in the next section we review related work primarily considering other systems which involve active cameras. In section III we describe the overall system architecture, and specific implementation details for each of the modules are considered in section IV. We show preliminary results from an actual implementation of the system in section $\mathrm{V}$, and conclude with a summary of our current and future research directions.

\section{RELATED WORK}

There has been a significant body of research published on distributed multi-camera surveillance systems in recent years. Since in this work we are concerned with a system that supports active cameras - i.e. cameras which can zoom, pan and tilt - the following review concentrates on other systems employing similar devices.

By far the most common means to use active cameras is in the context of a master-slave-configuration, in which a set of dedicated, static supervisor cameras makes wide area observations and coordinates control of the active sensing 
parts [1], [2], [3]. A disadvantage of supervisor cameras is the need for a mapping of image contents to the active camera, which has to be obtained from restricted camera placements, movements or temporally extended observations [4], [5], [6].

Methods exist which obtain an explicit calibration from target movements [7], [8], or a mapping of world, or gaze coordinates to gaze parameters of the slave. For this, [6] and [2] use a single moving target, whereas [5] uses long term observations. These efforts can be circumvented by restricted camera placements [3], [4]. In contrast, systems without supervisor cameras have to rely on heuristics to observe the scene [9], [10].

Most of the systems above do not require the integration of data from multiple sources into a common coordinate system. In particular, positional data obtained by the active camera is usually not fed back into the system, except for high resolution image data obtained [6], [3], [1]. Similar to [2], [1], our system is also fully calibrated, since we aim at integration of data from all participating sensors in a single world coordinate system. This strategy is akin to [2], but with higher degree of flexibility. Whereas the active vision part uses a local controller strategy close to their " $\mu$ Saccade", the resulting data in our system is fed back to the database. This includes higher layers for more abstract reasoning, not only on the trajectory and visibility of the single target under scrutiny.

For data exchange in multi camera systems, two common aspects of system design - efficiency and modularity - need to be balanced. The modularity aspect becomes inescapable once the system is distributed among different machines.

Since active vision systems need shorter response times, they often make use of less standardised methods, such as sockets [11], or restrain themselves to multi-threaded applications on single machines [1], [4], [3]. A solution could be XML [12] because of the human-readability and versatility of this format. Unfortunately, the XML standard trades efficiency for flexibility, thus it is seldom the first choice for active vision systems. A promising alternative is modularisation based on agents presented in [13], but the actual implementation of the communication is unfortunately not touched.

A multi camera surveillance system that also proposes a database for communication is presented in [14]. The authors present a multi-camera surveillance system with over twenty video cameras in a realistic, long-term setting. Real-time video analysis (tracking and event detection) results are stored online in a central database. The database supports parallel inserts as well as queries to the data. However, no information is given about the latency of the whole system.

\section{System ARChiteCtURE}

Our aim is to develop a system comprising distributed static and active cameras (with more or less overlapping fields of view), supporting asynchronous real-time operations to enhance the detection and tracking of targets. Our architecture is based on a central supervising unit which communicates with a set of semi-autonomous visual tracking processes.

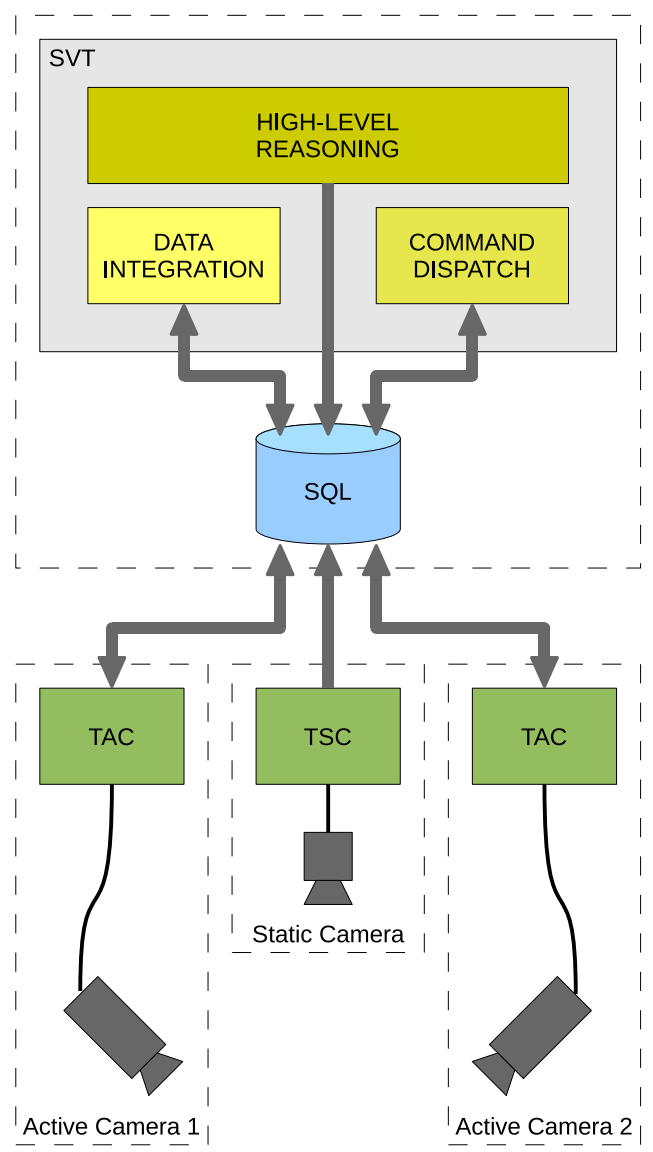

Fig. 1. System architecture.

Ultimately our objective for this supervisor process is to assimilate tracking results from various cameras, deal with assignments of cameras to targets, and to implement a layer between low-level primarily data-driven processes, and higherlevel processes concerned with scene interpretation and representation and learning of ontologies, etc.

In the present work, however, we are concerned only with the supervisor, the data-driven, low-level processes, and the means of communication. Interprocess communications are mediated by the supervisor, and effected using an SQL database. The database provides a natural means by which messages can be passed between processes, in an architecture independent fashion, simply by writing to and reading from database tables/records, without the need to implement a potentially complex asynchronous communications protocol. It further provides an automatic means to archive data.

A schematic of the system is shown in Fig. 1. In summary, the key components of our architecture are the following ones:

- Tracker with static camera (TSC), provided with a wideangle lens;

- Tracker with active camera (TAC), comprising a firewire camera with integrated zoom lens (SONY DFW-VL500 and IMAGINGSOURCE DFK 21BF04-Z2) and a Directed Perception DTU-D46 pan-tilt unit;

- Supervisor tracker module (SVT) dealing with data inte- 
gration, command dispatch and high-level reasoning;

- SQL database shared by the above three components; the present implementation comprises 4 tables: one for observations/estimations generated by the trackers, one for high-level commands sent by the SVT, another one for stabilized images produced by the TAC, and finally one to store calibration information for all the trackers.

Each tracker acts as an independent node with its own processing unit connected to the systems via TCP/IP and communicating by means of SQL queries. The SVT runs on a linux-based machine with a MySQL server. All the units are synchronized using the standard Network Time Protocol (NTP).

The basic scheme of communication among the trackers, the database, and the supervisor module is illustrated in Fig. 2 and described next.

1) First, in an offline and one-off procedure, both cameras are calibrated (section IV-B) with the calibration data stored in the common repository.

2) The TSC starts retrieving information about the positions of the tracked targets, in form of regions of interest (ROI). The position of each target and its ROI is stored for each time step into an Observation Table.

3) The supervising module continuously reads the new information stored in the Observation Table. Using the calibration data and an assumption that the face appears near the top of the bounding box, the module computes an approximate set of world coordinates for the face/head of the target. These data are then stored together with the ID of the target in the same table.

4) The TAC tracker receives the commands from the SVT through the Command Table and reads the relative information being stored into the Observation Table, thus obtaining an approximated configuration of pan, tilt, zoom parameters, so that the initial position of the face can be predicted to initialize the tracking. Stabilized face images of the target, independently tracked by the TAC, are finally stored in the Image Table.

\section{IMPLEMENTATION}

\section{A. Passive Visual Sensing}

The static camera of our system is used for real-time human detection on wide-angle image sequences. Two different solutions have been implemented, one using a segmentation-based algorithm by Roth et al. [15] for an initial prototype of the system, and another one based on the Lehigh Omnidirectional Tracking System (LOTS) [16], which is part instead of a fixed installation covering a large public area. As explained next, the use of two different solutions depends mainly on the particular location and orientation of the static camera, but it shows also that the system architecture can easily adapt to different kind of cameras and tracking algorithms.

The first implementation consists in a multi-object tracker that performs a per-pixel classification, assigning every pixel to one of the different objects being tracked, including the static

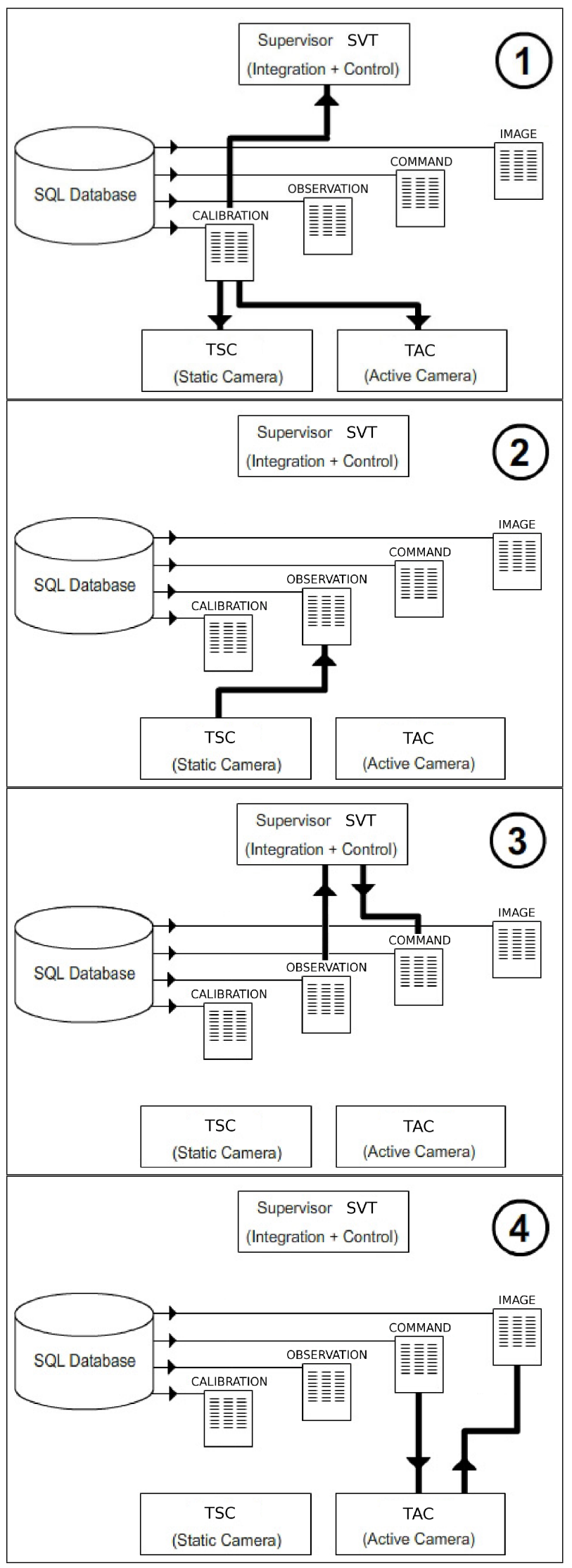

Fig. 2. Steps of the communication protocol between the TSC and the TAC to get stabilized images of the target detected by the TSC. 


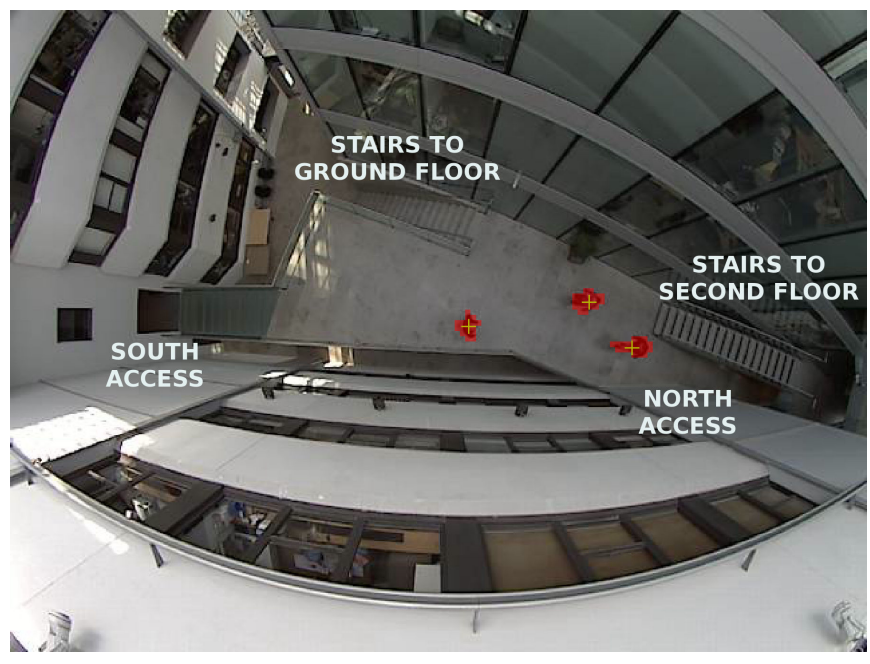

Fig. 3. People detection using LOTS background subtraction. The centroid of each red cluster, corresponding to a human target, is marked with a cross.

background. The classification is based on the probability that a given pixel belongs to one of the objects given its specific color and position. These object probabilities are determined on the basis of two components. First, the observed pixel values are compared to learned appearance models of the objects, in order to yield an indication of how similar observed colors are to these models. The appearance models use Gaussian mixtures in RGB color space with a single Gaussian per-pixel for the background and multiple Gaussians for the foreground models, and are learned and updated continuously. To handle occlusions, the lack of image depth information is partially compensated by assuming a horizontal and upright camera orientation, as well as a planar floor. The reader should refer to [15] for further information.

To detect people in the large atrium shown in Fig. 3 instead, we use an implementation of the LOTS algorithm as described in [17]. This is based on a background subtraction technique that uses two gray-scale images (of the background) and two per-pixel thresholds. The latter treat each pixel differently, allowing the detector to be robust to localized noise in lowsize image regions, and evolve according to a pixel label provided by a light version of the traditional connected component analysis. Small adjacent regions detected by LOTS are clustered together, and their centroid calculated to give the target position. The background subtraction well suits our current installation of the static camera because the targets, being observed from the top, do not overlap in this case (see Fig. 3). Details of the original LOTS algorithm can be found in [16].

\section{B. Calibration}

To enable the supervisor to provide demands for the active camera based on measurements from the static camera, both were calibrated in a common world coordinate frame. The intrinsic and extrinsic parameters of the static camera were determined in standard fashion using multiple images of planar checkerboard pattern, and yields among other things the position of a world ground-plane $(z=0)$.

Since the active camera is used for fixated closed-loop tracking, there is no need for accurate intrinsic calibration (though focal-length does play a role as a closed-loop gain in the system). The active camera is calibrated to the world frame via a series of fixations of known 3D points. The mapping from these points, or visual rays, to the joint-angles of the pan-tilt unit is given succinctly by a 3D-2D projectivity, where the assumption that the centre of rotation of the device is close to the optical centre of the camera enables us to treat the active camera as a "projective pointing device" [18].

At each time step the TSC tracker provides the image coordinates and the bounding box of each detected target. Using the assumption that the targets are in contact with the ground-plane, and the face is a fixed distance above the ground (in this work $1.7 \mathrm{~m}$ ), the 3D coordinates of the target face/head are computed by the supervisor module and stored in the Observation Table.

The accuracy of the extrinsic calibration has an effect on the relative success of what amounts to hand over from the static camera to the active camera. However once the active camera is tracking under closed-loop control, it can continue to do so independently of the static camera view.

\section{Active Visual Tracking}

The active tracker module periodically polls the database to determine if there is a new demand from the supervisor. If a new demand is present, the pan-tilt unit is immediately commanded to the appropriate angle, and the zoom level of the camera set to a value such that, based on the estimated depth of the target, the face will occupy a fixed fraction of the field of view.

A face detection module, based on the OpenCV implementation of the Viola-Jones boosted face detection algorithm [19] locates the face in the image when the pan-tilt demand is satisfied and is used to initialise a region-based tracker. The latter uses a level-set to simultaneously track the image position, rotation and scale of the target while segmenting it from the background [20]. This tracker is fast (run-time of 20-30ms) and robust, and provides a smooth demand to a local velocity control regime. The controller runs at the same rate as the visual processing and employs a piece-wise linear proportional control signal as used in [21].

Zoom control on the active camera is slow, and subject to significant latencies. The scale of the target, as computed by the level-set tracker, is used to control the zoom to keep the face to a fixed size, chosen heuristically as a balance between high-resolution and the ability of the PTZ device to keep the target in the field of view.

\section{System Supervision}

The supervisor module is responsible for the data integration , command dispatch and high-level reasoning of the system. As anticipated in Fig. 1, this can be thought as a hierarchical structure of semi-independent processes where the high-level 
reasoning part supervises the other two using bi-directional communication, via SQL queries, for information retrieval and control.

The main purpose of the data integration process is to collect sensor observations from one or more cameras and generate proper trajectories of the current targets, which can be used for high-level reasoning and for the active cameras. In the current system, this is implemented as a multi-target tracker based on Kalman filter and Nearest Neighbour data association using a constant-velocity model [22], [23]. The multi-target tracker reads from the Observation Table the 2D world coordinates of the targets provided by the static camera, and then writes the relative estimates (2D positions and velocity) into the same table. In this way, it is possible to identify the trajectory of a target, as a sequence of estimates with a unique ID, from the anonymous detections provided by the static camera.

The command dispatch process is responsible for the correct interpretation and delivery of the high-level commands. These are sent to the destination cameras through the Command Table, as explained in IV-E, in some cases together also with additional arguments (e.g. the ID of a target to follow).

Finally, the high-level reasoning plays the double role of high-level reasoning process and controller. At the moment, this is achieved partly using a rule-based approach, and partly with a real-time implementation of an inference engine based on fuzzy metric-temporal logic [24]. The high-level reasoner selects the best TAC to use for tracking a person according to the his/her location and orientation, in order to increase the chances of capturing high-resolution images of faces.

\section{E. Data Exchange via SQL Tables}

Each component of the system (TSC, TAC and SVT) sends the local observation/estimation to the Observation Table. The structure of this table has been defined thus to simplify the communication between tracker clients and supervisor, and includes records containing the following 23 fields:

\begin{tabular}{|l|l|}
\hline timestamp, source_id, target_id & (identification) \\
\hline top, left, bottom, right & (2D bounding box) \\
\hline$\Sigma_{11}, \Sigma_{12}, \Sigma_{22}$ & (2D uncertainty) \\
\hline$x, y, z$ & (3D coordinates) \\
\hline$\Sigma_{x x}, \Sigma_{x y}, \Sigma_{x z}, \Sigma_{y y}, \Sigma_{y z}, \Sigma_{z z}$ & (3D uncertainty) \\
\hline$v_{x}, v_{y}, v_{z}$ & (3D velocities) \\
\hline data & (general purpose) \\
\hline
\end{tabular}

Basically, the first three fields contain the time of the current information, the ID of the source device (camera) and that one of the target being followed. The 2D bounding box of the target, with respect to the current camera's frame, is then recorded together with the upper triangular entries of the relative (symmetric) covariance matrix. The following fields contain the 3D position of the target, its velocity and its covariance matrix (upper triangular entries). This information depends on the extrinsic parameters of the camera and, therefore, on the pan/tilt orientation in case of active camera. The last field is left for the possible transmission of additional data.
Commands are sent by the supervisor module to the TAC through the Command Table, the structure of which is defined as follows:

\begin{tabular}{|l|l|}
\hline timestamp, destination_id, command_id & (identification) \\
\hline data & (arguments) \\
\hline
\end{tabular}

The destination TAC of the command is specified in the "destination_id" field. Commands are read by the TAC periodically polling the table. At the moment, the list of implemented commands includes the following 8 ones (but this can be easily extended):

1) CAPTURE_CALIBRATION_IMAGE - capture image for calibration setup

2) RELOAD_CALIBRATION_DATA - load calibration settings from database

3) GO_TO_LOCATION - point camera towards a desired 3D point

4) FOLLOW_TARGET - point camera towards a target in the Observation Table

5) TRACK_TARGET - point camera towards a target and instantiate a local tracker

6) STOP_TRACKING - stop current target tracking

7) STOP_MOTION - stop every activity

8) SET_DEFAULT_OBSERVATION_VOLUME - set default view while idle

Finally, the face images captured by TAC while tracking are stored in the Image Table in records defined as follows:

\begin{tabular}{|l|l|}
\hline timestamp, source_id, target_id & (identification) \\
\hline data, format & (image) \\
\hline
\end{tabular}

where "data" contains the binary image and "format" specifies the whether it is MONO, RGB, etc.

\section{RESUlts}

Several experiments have been run on an initial prototype of system, shown in Fig. 4(a), and on the final installation covering a large atrium of our department, as illustrated in Fig. 4(b). The first one includes a static camera, mounted on the ceiling, and an active camera on a tripod. The second comprises a static camera in the middle of the atrium and two active cameras on the north and south sides.

\section{A. System Latency}

To begin, we consider the performance of the MySQL database and demonstrate the fact that this represents not only a means to archive tracking data, but also an effective way to implement interprocess data exchange in a timely manner.

Table I and II show transaction timings for 4000 data entries and retrievals with MySQL running on a Dual-core Intel processor under Linux. They illustrate in particular the latency introduced by the database, remotely accessed via TCP/IP, from the moment the TSC inserts an observation until the TAC receives the relative command (steps 2, 3 and 4 in Fig. 1). The first table shows the transaction timings in the simplest case, when only the TSC and one TAC are used, while the second 


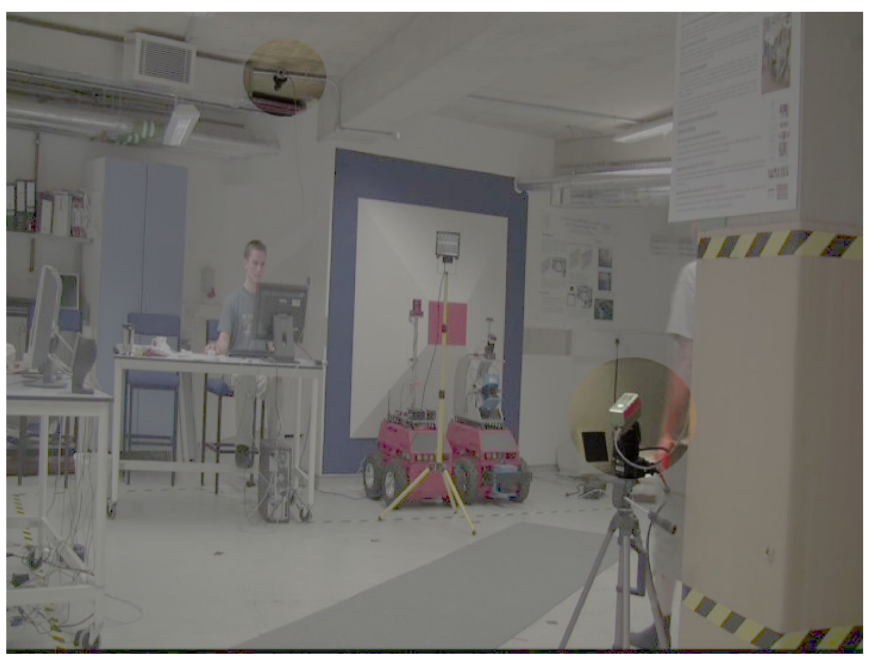

(a)

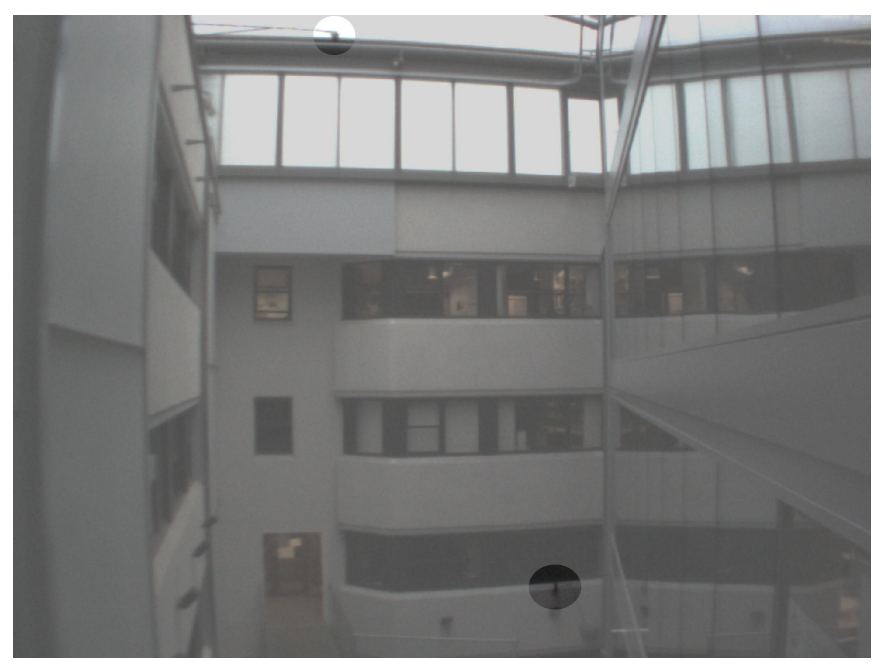

(b)

Fig. 4. (a) System setup for the initial prototype (active camera on the bottom right) and (b) for the final installation in a large atrium (static camera on the top, active on the bottom).

\begin{tabular}{|l|c|c|c|c|}
\hline One TAC & Min & Max & Mean & SD \\
\hline \hline TSC $\rightarrow$ TAC & 1.7 & 59.9 & 3.7 & 3.2 \\
\hline \multicolumn{5}{|c}{ TABLE I }
\end{tabular}

TIMINGS IN MS FOR 4000 DATA ENTRIES-RETRIEVALS WITH ONE TAC.

\begin{tabular}{|l|c|c|c|c|}
\hline Two TACs & Min & Max & Mean & SD \\
\hline \hline TSC $\rightarrow$ TAC1 & 1.5 & 53.4 & 3.2 & 1.6 \\
\hline TSC $\rightarrow$ TAC2 & 1.8 & 52.1 & 3.6 & 1.5 \\
\hline
\end{tabular}

TABLE II

TIMINGS IN MS FOR 4000 DATA ENTRIES-RETRIEVALS WITH TWO TACS.

refers to the case with two TACs simultaneously accepting commands.

These results are significant because they demonstrate that the round-trip time for a message from the TSC to the TAC is sufficiently small as to be negligible compared to typical camera framerates, even when the database contains millions of entries (in this case, more than 60 million for the Observation Table and 18 million for the Command Table). As shown by our experiments, indeed, the delay is in average one order of magnitude less than the $30 \mathrm{~Hz}$ of a standard camera.

\section{B. Data Archiving}

SQL provides an effective tool to monitor human activities and record large amounts of interesting data to be later analyzed. In this case, we were focusing on the typical trajectories followed by people when crossing the surveilled atrium.

A trajectory is simply a sequence of $2 \mathrm{D}$ coordinates labelled with the ID of the relative person. Fig. 5 shows a series of 179 trajectories, recorded during an experiment 4 hours long and extracted from almost 60000 labelled coordinates, which were generated by the SVT using panoramic observations from the TSC.
This is just an example of the potential benefits provided by the proposed architecture. Many other details are available for each coordinate in the database (i.e. timestamp, velocity, etc.), from which lots of information can be extracted.

\section{Multi-Resolution Surveillance}

Fig. 6 shows a series of stills from an extended sequence of operation in which the supervisor initially instructs the active camera to track one of the targets and maintain his face at the same size (first two frames, $t=20 \mathrm{~s}$ and $t=25 \mathrm{~s}$ ). Note the stable view of the face in spite of motion blur in the background induced by the camera motion. After tracking and recording stabilised images of the first person's face for a period of time, the supervisor decides to switch attention to a second more recently acquired target. After the demand is sent to the camera, this moves to the new target, acquires it and finally tracks it in closed-loop control (last two frames, $t=42 s$ and $t=43 s$ ).

An example of target acquisition and tracking at long distances is shown in Fig. 7. In the first two frames, the white box outlines the latest target position as received from the database. Note the motion blur in the first frame - Fig. 7(a), induced by higher velocity demands for initial centering of the target. When the target is roughly centered, the camera follows at lower speeds, at the same time zoom is adjusted to maximise the area occupied by the target. Once the target is detected - Fig. 7(b), the active tracker is initialised and zoom increased to maximum - Fig. 7(c)-7(f). Finally, the maximum zoom is reached, and the target face is kept and tracked at high resolution. The target's positional uncertainty obtained from the database has to be overestimated to take into account the errors due to calibration and odometry delay of the pan/tilt head and camera. 


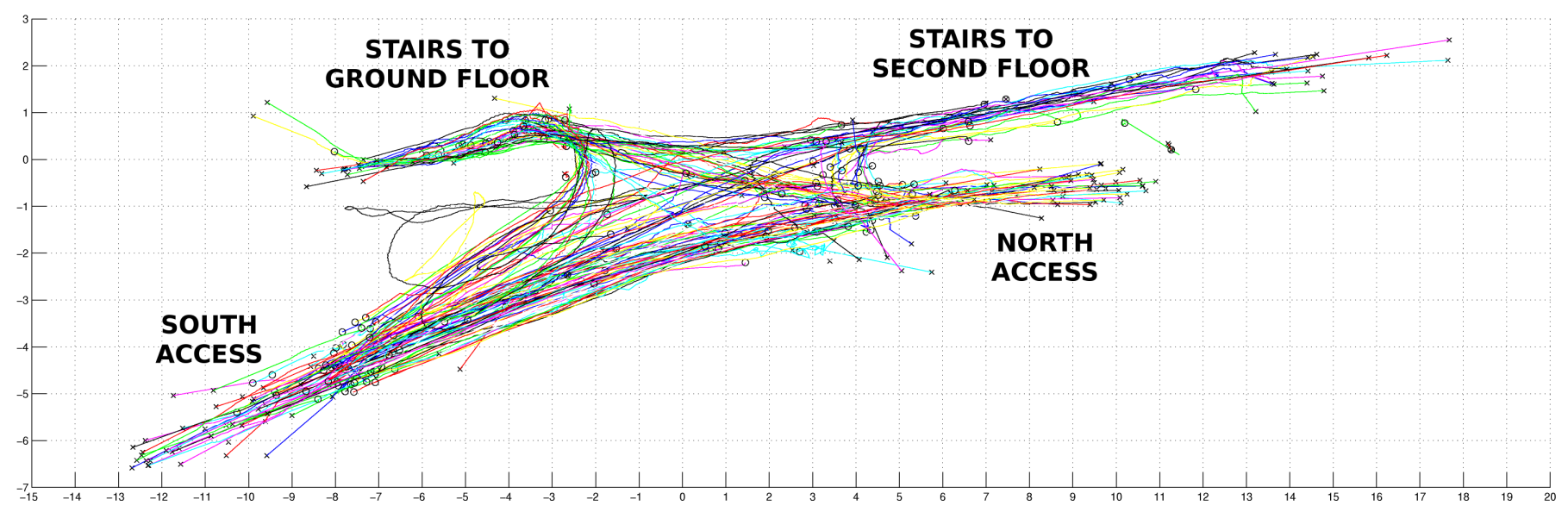

Fig. 5. Trajectories of people walking in the atrium (grid $1 \times 1 \mathrm{~m}^{2}$ ) during a 4 hour period. Every trajectory has a start- and an end-point, marked with a cross and a circle respectively.

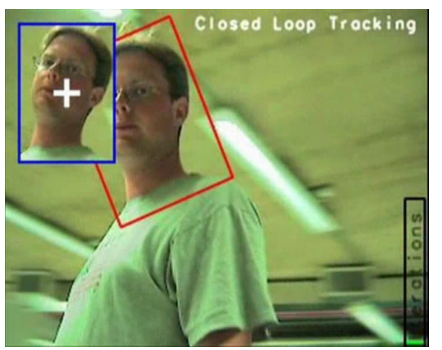

(a) $t=20 \mathrm{~s}-$ target acquired

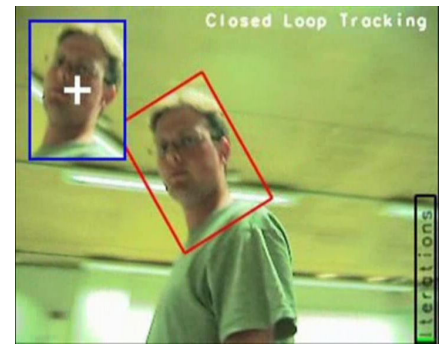

(b) $t=25 \mathrm{~s}-$ follow target

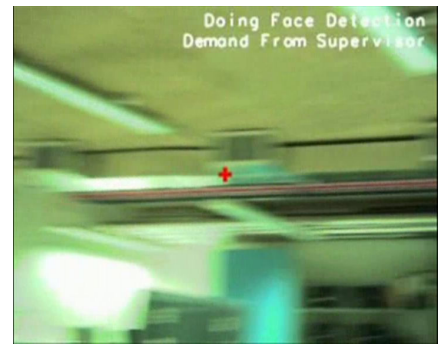

(c) $t=42 s-$ pan

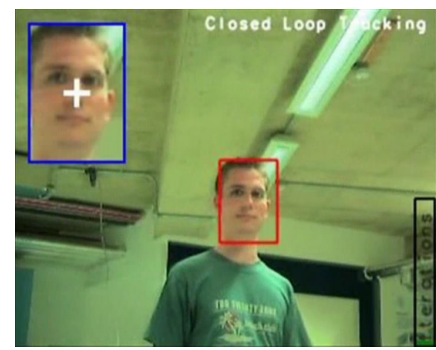

(d) $t=45 \mathrm{~s}-$ new target acquired

Fig. 6. Stills from an extended sequence of multi-resolution tracking, where the active camera is switching from one target to another, as demanded by the supervisor tracker.

\section{CONCLUSIONS}

We have presented an architecture for a distributed camera system and described implementation details for a real system comprising a static camera and two active cameras. While there is little novelty in the algorithms used, we argue that there is value in the architectural decisions we have made, most notably the use of a central SQL database used as both an archival repository, and a means to communicate between loosely connected visual processes.

There remains much to do to prove the scalability of the architecture, both in terms of numbers of cameras, and in terms of robust operation over extended periods. Presently the supervision module is rule-based, and the assignment of cameras to tasks is made in a completely rigid manner, once and forever. This is not imposed by the architecture, and we are in the process of implementing the technique of [10] which provides a means to regulate zoom as a balance between possible target lock, and obtaining high resolution images of the target.

\section{ACKNOWLEDGMENT}

This work was supported by the EU FP6 grant number IST027110 for the HERMES project - http://www.hermes-project.eu.

\section{REFERENCES}

[1] A. Hampapur, S. Pankanti, A. Senior, Y.-L. Tian, L. Brown, and R. Bolle, "Face cataloger: Multi-scale imaging for relating identity to location," in Proc. of the IEEE Conf. on Advanced Video and Signal Based Surveillance, Washington, DC, USA, 2003, pp. 13-20.

[2] P. Peixoto, J. Batista, and H. Araujo, "A surveillance system combining peripheral and foveated motion tracking," Pattern Recognition, 1998. Proc.. Fourteenth Int. Conf. on, vol. 1, pp. 574-577 vol.1, Aug 1998.

[3] J. H. Elder, S. J. Prince, Y. Hou, M. Sizintsev, and E. Olevskiy, "Preattentive and attentive detection of humans in wide-field scenes," Int. $J$. Comput. Vision, vol. 72, no. 1, pp. 47-66, 2007.

[4] F. Bashir and F. Porikli, "Collaborative tracking of objects in EPTZ cameras," in Visual Communications and Image Processing 2007, C. W. Chen, D. Schonfeld, and J. Luo, Eds., vol. 6508. SPIE, 2007.

[5] U. M. Erdem and S. Sclaroff, "Look there! predicting where to look for motion in an active camera network," in IEEE Conf. on Advanced Video and Signal Based Surveillance, 2005, pp. 105-110.

[6] F. Z. Qureshi and D. Terzopoulos, "Surveillance in virtual reality: System design and multi-camera control," in IEEE Conf. on Computer Vision and Pattern Recognition, 2007, pp. 1-8.

[7] T. Chen, A. Del Bimbo, F. Pernici, and G. Serra, "Accurate selfcalibration of two cameras by observations of a moving person on a ground plane," in Proc. of IEEE Int. Conf. on Advanced Video and Signal Based Surveillance, 2007, pp. 129-134.

[8] N. Krahnstoever and P. Mendon ca, "Autocalibration from tracks of walking people," in British Machine Vision Conf. (Vol. I), 2006, pp. $107-116$.

[9] J. Davis, A. Morison, and D. Woods, "An adaptive focus-of-attention model for video surveillance and monitoring," Machine Vision and Applications, vol. 18, no. 1, pp. 41-64, 2007.

[10] E. Sommerlade and I. Reid, "Information theoretic active scene ex- 


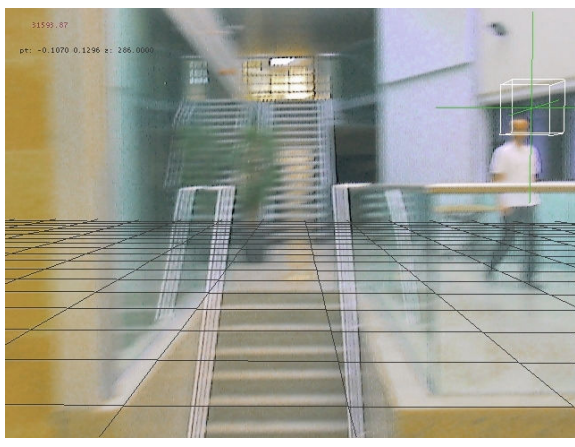

(a) Frame 25 - pan

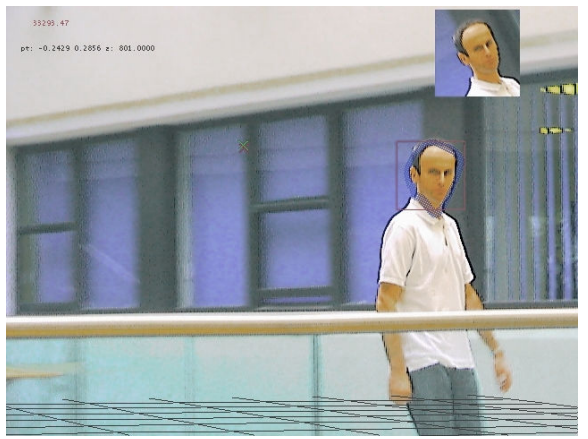

(d) Frame 67 - zoom in

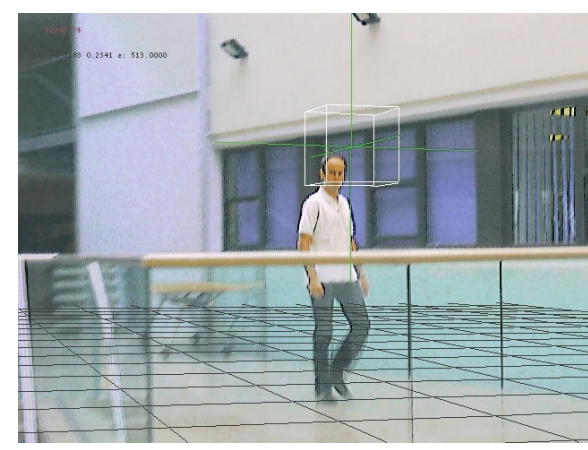

(b) Frame 46 - detection

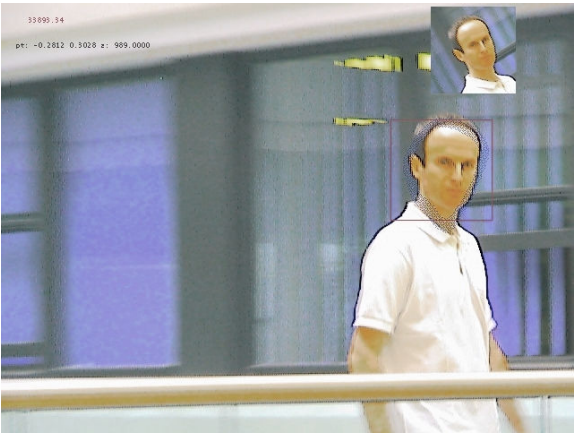

(e) Frame 83 - zoom in

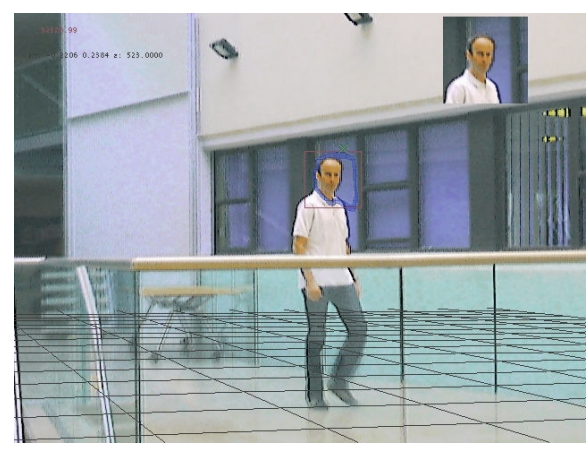

(c) Frame 47 - acquisition

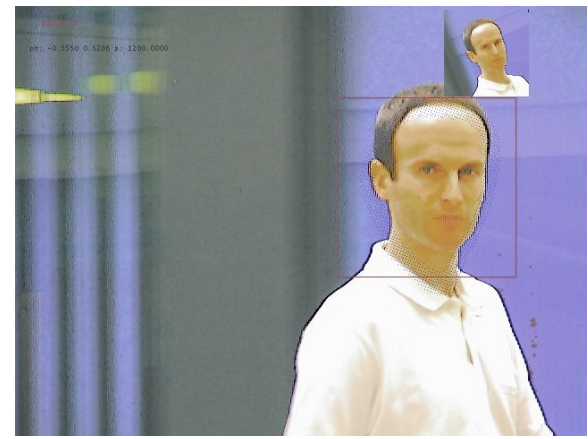

(f) Frame $115-$ max zoom

Fig. 7. Multi-resolution tracking over long distances. Zoom factors are changed from 1 to 2.5 in frame $25,4.5$ in frames 46 and 47 , up to 10 in frame 115 . Note the stabilised closeup image in the upper right corner once the target is acquired, and which is stored in the database. To speed up initial acquisition of the target, the active tracker is not always run until full convergence (see face boundary in 47). This can result in subsequent rotation of the closeup image.

ploration," in Proc. IEEE Computer Vision and Pattern Recognition (CVPR), 2008, pp. 1-7.

[11] L. Marchesotti, L. Marcenaro, and C. Regazzoni, "Dual camera system for face detection in unconstrained environments," in Int. Conf. on Image Processing (ICIP), vol. 1, 2003, pp. 681-684.

[12] D. Thirde, M. Borg, J. Ferryman, F. Fusier, V. Valentin, F. Bremond, and M. Thonnat, "A real-time scene understanding system for airport apron monitoring," in IEEE Int. Conf. on Computer Vision Systems (ICVS), 2006, p. 26.

[13] P. Remagnino, A. I. Shihab, and G. A. Jones, "Distributed intelligence for multi-camera visual surveillance," Pattern Recognition, vol. 37, no. 4, pp. 675-689, 2004

[14] A. Girgensohn, D. Kimber, J. Vaughan, T. Yang, F. Shipman, T. Turner, E. Rieffel, L. Wilcox, F. Chen, and T. Dunnigan, "Dots: support for effective video surveillance," in Proc. of the 15th Int. Conf. on Multimedia, 2007, pp. 423-432.

[15] D. Roth, E. Koller-Meier, and L. V. Gool, "Multi-object tracking driven event detection for evaluation," in AREA workshop @ ACM Multimedia, October 2008, in press.

[16] T. Boult, R. Micheals, X. Gao, and M. Eckmann, "Into the woods: visual surveillance of noncooperative and camouflaged targets in complex outdoor settings," Proceedings of the IEEE, vol. 89, no. 10, pp. 13821402,2001
[17] D. Hall, J. Nascimento, P. Ribeiro, E. Andrade, P. Moreno, S. Pesnel, T. List, R. Emonet, R. B. Fisher, J. S. Victor, and J. L. Crowley, "Comparison of target detection algorithms using adaptive background models," in Proc. of the 14th Int. Conf. on Computer Communications and Networks, 2005, pp. 113-120.

[18] A. J. Davison, I. D. Reid, and D. W. Murray, "The active camera as a projective pointing device," in Proc. of the 6th British Conf. on Machine vision (Vol. 2), 1995, pp. 453-462.

[19] P. Viola and M. J. Jones, "Rapid object detection using a boosted cascade of simple features." in IEEE Conf. on Computer Vision and Pattern Recognition, Kauai, HI, USA, 2001, pp. 511-518.

[20] C. Bibby and I. Reid, "Robust real-time visual tracking using pixel-wise posteriors," in Proc. of the 10th European Conf. on Computer Vision (ECCV), 2008, pp. 831-844.

[21] — "Visual tracking at sea," in Proc. of the IEEE Int. Conf. on Robotics and Automation (ICRA), April 2005, pp. 1841-1846.

[22] N. Bellotto and H. Hu, "People Tracking with a Mobile Robot: a Comparison of Kalman and Particle Filters," in Proc. of the 13th IASTED Int. Conf. on Robotics and Applications, 2007, pp. 388-393.

[23] Y. Bar-Shalom and X. R. Li, Multitarget-Multisensor Tracking: Principles and Techniques. Y. Bar-Shalom, 1995.

[24] H.-H. Nagel, "Steps toward a cognitive vision system," AI Magazine, vol. 25 , no. 2, pp. 31-50, 2004. 